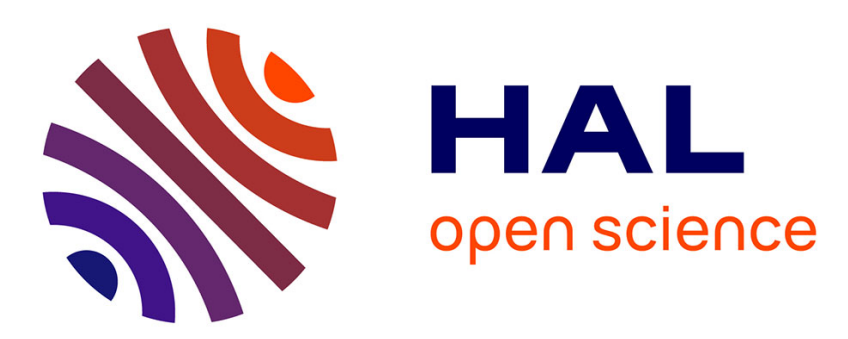

\title{
Quand la constellation exorcise la peur du ciel : histoire d'un impensable astronomique chez Émile Zola Émilie Piton-Foucault
}

\section{To cite this version:}

Émilie Piton-Foucault. Quand la constellation exorcise la peur du ciel : histoire d'un impensable astronomique chez Émile Zola. Romantisme: la revue du dix-neuvième siècle, 2014, "L'Astronomie", 166 (4), pp.53-64. 10.3917/rom.166.0053 . hal-01673515

\section{HAL Id: hal-01673515 \\ https://hal.univ-rennes2.fr/hal-01673515}

Submitted on 30 Dec 2017

HAL is a multi-disciplinary open access archive for the deposit and dissemination of scientific research documents, whether they are published or not. The documents may come from teaching and research institutions in France or abroad, or from public or private research centers.
L'archive ouverte pluridisciplinaire HAL, est destinée au dépôt et à la diffusion de documents scientifiques de niveau recherche, publiés ou non, émanant des établissements d'enseignement et de recherche français ou étrangers, des laboratoires publics ou privés. 
QUAND LA CONSTELLATION EXORCISE LA PEUR DU CIEL : HISTOIRE D'UN IMPENSABLE ASTRONOMIQUE CHEZ ÉMILE ZOLA

Émilie Piton-Foucault

Armand Colin | « Romantisme »

2014/4 n 166 | pages 53 à 64

ISSN 0048-8593

ISBN 9782200929091

Article disponible en ligne à l'adresse :

https://www.cairn.info/revue-romantisme-2014-4-page-53.htm

\section{Pour citer cet article :}

Émilie Piton-Foucault, «Quand la constellation exorcise la peur du ciel : histoire d'un impensable astronomique chez Émile Zola », Romantisme 2014/4 ( $\left.\mathrm{n}^{\circ} 166\right)$, p. 53-64.

DOI 10.3917/rom.166.0053

Distribution électronique Cairn.info pour Armand Colin.

(C) Armand Colin. Tous droits réservés pour tous pays.

La reproduction ou représentation de cet article, notamment par photocopie, n'est autorisée que dans les limites des conditions générales d'utilisation du site ou, le cas échéant, des conditions générales de la licence souscrite par votre établissement. Toute autre reproduction ou représentation, en tout ou partie, sous quelque forme et de quelque manière que ce soit, est interdite sauf accord préalable et écrit de l'éditeur, en dehors des cas prévus par la législation en vigueur en France. Il est précisé que son stockage dans une base de données est également interdit. 


\section{Quand la constellation exorcise la peur du ciel : histoire d'un impensable astronomique chez Émile Zola}

Dès 1864 et de façon continue jusqu'en 1869, Émile Zola évoque sa fascination pour l'astronomie en se faisant l'écho des travaux de vulgarisation d'Amédée Guillemin. Théories de Kepler et de Laplace, évocation de la composition physique du soleil..., son discours manifeste une réelle appropriation des ouvrages, qu'il s'excuse de critiquer comme un néophyte, mais comme un néophyte passionné que la clarté pédagogique de Guillemin pousse à partager son enthousiasme ${ }^{1}$ : "La poésie de son livre est haute et grave ; elle naît autant de la nature du sujet que du style de l'écrivain. Ce style est ce qu'il devait être : sobre, simple, peignant d'un mot et ouvrant l'infini sans emphase ${ }^{2}$. "Faut-il voir là une critique en creux des débordements stylistiques de Camille Flammarion, qui s'affirme alors comme le vulgarisateur par excellence des observations astronomiques? Flammarion ne devait guère goûter de son côté l'œuvre de Zola, qui semble parfaitement correspondre à cette pique assassine présente dans son Astronomie populaire de 1880 - alors même que l'ouvrage paraît grâce aux bénéfices engrangés par L'Assommoir : "L'aspiration de l'esprit humain vers la Vérité, vers la conception du beau dans la nature, vers le progrès indéfini, constituant le fait le plus caractéristique de l'histoire de l'humanité, n'est-il pas singulier de voir, à notre époque, un écrivain consacrer sa vie entière à essayer de démontrer que "l'humanité, c'est de la viande ${ }^{3 "}$ ?"

De nombreux éléments rapprochent cependant les deux hommes. La conquête de la vérité par la science évoquée en clôture de l'Astronomie populaire offre ainsi un curieux mimétisme avec le discours radical tenu par Zola dans les années 1890 :

Il n'y a plus aujourd'hui de science cachée; les chemins du ciel sont ouverts pour tout le monde; chacun peut étudier la réalité splendide au sein de laquelle la plupart des hommes ont vécu jusqu'ici comme des aveugles. L'Astronomie est

1. « [On trouvera] en moi un critique des plus ignorants. Mais [...] comme j'ai mille choses à vous dire [...], je vais vous les dire tranquillement, au risque de faire rire la galerie. À coup sûr, les auteurs seront plus touchés de ma franchise, même de mes sottises, filles d'une naïve admiration, que des éloges aigres-doux de leurs confrères en science » (Émile Zola, article paru dans L'Écho du Nord des 26 et 27 décembre 1864, Euvres complètes, Henri Mitterand (éd.), Paris, Tchou, « Cercle du livre précieux », 1966-1969, tome X, p. 321-322)

2. Ibid., p. 328. Voir aussi l'article paru dans Le Gaulois du 24 août 1869 : «Ce dont je le remercie surtout, c'est de ne pas chercher à enrubanner la science et à la fleurir d'anecdotes » (ibid., p. 900).

3. Camille Flammarion, Astronomie populaire, Paris, Flammarion, 2009 [1880], tome II, p. 593. 
la vraie science intégrale, et elle est aussi la vraie religion de l'avenir [...]. Nous sommes à une époque où les erreurs de l'ignorance, les fantômes de la nuit, les songes de l'enfance humaine doivent disparaître ; l'aurore répand sa pure lumière ; le soleil se lève sur l'humanité éveillée ; tenons-nous debout devant le ciel et n'ayons désormais qu'une seule et même devise : LE PROGRÈS PAR LA SCIENCE ${ }^{4}$ !

Un tel propos ne dépareillerait pas au sein des envolées lyriques du Docteur Pascal, décrivant en clôture des Rougon-Macquart la conquête scientifique de la vérité contre le mensonge rassurant de la foi. Or, dans cette campagne prophétisant la mort « darwinienne " de la religion, le motif astronomique prend chez Zola un rôle crucial. Dans un article de 1896, le modèle de Galilée est ainsi l'embrayeur de la démonstration du recul progressif et inéluctable du catholicisme :

Pour moi, le catholicisme est condamné à disparaître devant la science, parce qu'elle a déjà ruiné ses dogmes et qu'elle les ruinera de plus en plus. [...] Est-ce que la science a jamais reculé ? C'est le catholicisme qui a toujours reculé devant elle, et qui sera forcé de reculer toujours. L'idée de Galilée, l'idée que la Terre tournait autour du Soleil a été châtiée comme un blasphème contre l'Écriture, et il faut bien que les prêtres eux-mêmes enseignent dans leur université que la Terre tourne. [...] Continuellement, la science forcera le catholicisme à reculer ainsi ${ }^{5}$.

Ce lien entre conquête de la vérité et astronomie, Zola le tisse dès sa première critique d'Amédée Guillemin : "Notre dernière pensée en fermant le volume a été que le ciel de Laplace est plus vaste, plus grandiose que le ciel de Dante ${ }^{6}$. "Chez le naturaliste, comme chez Flammarion qui reprend la même image ${ }^{7}$, le ciel se fait le support métaphorique idéal du nécessaire renversement des autorités; vidée de l'imagerie chrétienne, sa surface se déploie en un espace infini dédié à l'exploration scientifique :

Lorsque, après la lecture du livre de M. Guillemin, [...] on lit la création des sept jours, [...] l'horizon se rétrécit, l'égoïsme perce, ce n'est plus là qu'une fable dont l'être a bercé son orgueil. Aujourd'hui, les cieux sont dépeuplés, les mythologies sont mortes, les poèmes, si beaux qu'ils soient, perdent leur splendeur en face des vérités ${ }^{8}$.

Dans Le Docteur Pascal, le débat passionné opposant le médecin, double de l'écrivain, et la jeune Clotilde alors tentée par le mysticisme, est initié sous un ciel « immense, criblé d'étoiles, dans son infini de velours sombre ». Le docteur, parti à la recherche de sa nièce dans le jardin, finit par la retrouver par terre, " couchée sur le dos, les mains ramenées et serrées sous la nuque, la face vers le ciel ${ }^{9}$. " L'immensité de la nuit

4. Ibid., p. 563-564.

5. Émile Zola, « La science et le catholicisme », Euvres complètes, éd. citée, tome XIV, p. 839.

6. L'Écho du Nord, art. cité, p. 326.

7. «Élevons-nous comme lui vers les célestes hauteurs, non plus sur les ailes tremblantes de la foi, mais sur les fortes ailes de la science. » (Astronomie populaire, ouvr. cité, p. 356).

8. L'Écho du Nord, art. cité, p. 325.

9. Émile Zola, Le Docteur Pascal, Les Rougon-Macquart, Henri Mitterand et Armand Lanoux (éd.), Paris, Gallimard, « Pléiade », 1960-1967, tome V, p. 987-988. 
vient redoubler l'appel de l'infini mystique pour Clotilde, et donc représenter l'illusion et le mystère auxquels elle aspire à ce moment de l'intrigue. La contemplation des cieux devient le relais fictionnel d'un débat sur les espérances déçues du positivisme, où Zola répond aux arguments que lui oppose depuis plusieurs années Ferdinand Brunetière, réponse qu'il reprendra d'ailleurs dans des termes quasi-identiques dans un discours tenu à l'Assemblée des Étudiants de Paris en mai $1893^{10}$. Le lien entre la thématique astronomique et les enjeux du débat ne fait aucun doute lorsqu'on observe la construction du texte, qui se plaît à entrecouper les prises de position de chacun des deux protagonistes par la mention du ciel étoilé :

Et, de ses grands yeux immobiles, élargis et fixes, ses regards semblaient monter plus haut, parmi les étoiles. Elle était toute dans l'infini pur de ce ciel d'été, au milieu des astres. [...]

"Comme cela est étroit et borné, tout ce que tu sais, à côté de ce qu'il y a sûrement là-haut... [...] [E]ncore cent ans peut-être, et le ciel serait ouvert, nous serions face à face la vérité... Eh bien! les années marchent, et rien ne s'ouvre, et la vérité recule. [...] À [lire tes livres], il semble qu'on marche à la conquête de la terre et du ciel. Ils démolissent tout et ils font le serment de tout remplacer [...]. En tout cas, continua-t-elle, la science a fait table rase, la terre est nue, le ciel est vide, et qu'est-ce que tu veux que je devienne? [...]"

Un instant, ils se turent, sans un mouvement, les regards perdus parmi les milliards de mondes, qui luisaient au ciel sombre. Une étoile filante traversa d'un trait de flamme la constellation de Cassiopée. Et l'univers illuminé, là-haut, tournait lentement sur son axe, dans une splendeur sacrée. [...]

Alors, ils restèrent un instant face à face, muets et menaçants [...] ; et, sur leur tête, il semblait que le vaste ciel criblé d'étoiles eût pâli d'un frisson ${ }^{11}$.

Un modèle de la diffusion des avancées de son temps donc, que ce Zola chez qui le ciel se transforme comme chez Flammarion en symbole du progrès scientifique. Il y a cependant un monde entre ce discours explicite et son exploitation sur le plan littéraire. Derrière l'identité idéologique des discours et l'articulation manifeste du combat zolien au motif de l'infini étoilé, de sérieuses différences se font jour dans l'œuvre romanesque, livrant un témoignage de l'écart qui peut résider entre l'assimilation d'une science et sa réelle préhension par les individus du XIX ${ }^{\mathrm{e}}$ siècle. Dans le cas de Zola, le volontarisme de la quête scientifique ne résorbe nullement un rapport traumatique à la contemplation des astres.

\section{« L'HEUREUSE IGNORANCE DU LABOUREUR »}

L'astronomie est depuis toujours un vecteur d'imaginaire et de fantasme. Des voyages de Cyrano plaçant dans les mondes lunaire et solaire les désirs les plus libertins, à la projection par Flammarion de son rapport traumatique au corps et à la nourriture 
dans des mondes fictionnels où les êtres sont dépourvus de matérialité ${ }^{12}$, l'infini du ciel apparaît comme le réceptacle idéal de notre rapport à l'inconnu, quel que soit le discours scientifique professé par l'auteur. Sur ce point, Zola et Flammarion se rencontrent de nouveau mais pour se séparer définitivement. Que ce soit dans les œuvres fictionnelles ou les apartés de l'Astronomie populaire, l'exploration des cieux est toujours conçue par Flammarion comme un mode d'accès à une existence meilleure, idéale :

Concevons que nous voguions ainsi pendant un million d'années... Sommesnous aux confins de l'Univers visible ? Voici des immensités noires qu'il faut franchir... Mais là-bas de nouvelles étoiles s'allument au fond des cieux. Élançonsnous vers elles; atteignons-les. Nouveau million d'années : nouvelles révélations, nouvelles splendeurs étoilées! Nouveaux univers, nouveaux mondes, nouvelles terres, nouvelles humanités !... Eh quoi ! jamais de fin ? jamais d'horizon fermé ? jamais de voûte? jamais de ciel qui nous arrête ? [...] Nous sommes... au vestibule de l'infini $i^{13}$ !

Cette exaltation de l'infini devient vite le relais d'un rejet de l'existence terrestre, où Flammarion exprime son rapport trouble à la matérialité, caractéristique bassement animale dont l'homme ferait bien de se détacher : pauvres "mites " végétant par millions, l'homme ne trouverait pas mieux que de se réunir en " troupeaux " pour «s'égorger régulièrement " au lieu de s'élever aux « jouissances intellectuelles de l'art et de la science ${ }^{14}$ "! Rien de tout cela chez Zola, dont le rapport traumatique à l'infini vient au contraire parasiter le discours officiel sur la science. Ce parasitage commence dès les articles sur Guillemin, l'auteur revenant alors systématiquement sur la terreur que le ciel lui inspirait lorsqu'il était enfant :

Je me rappelle encore l'angoisse étrange, la profonde sensation de vertige qui me prit, lorsque, sur les bancs du collège, j'étudiais la cosmographie pour la première fois. Il me sembla que je me penchais sur un abîme, et que là, dans cette immensité, je voyais les astres se multiplier. La pensée humaine n'est pas faite pour la contemplation du ciel ; la folie viendrait vite si la raison s'arrêtait à l'idée d'un nombre infini de mondes dans l'infini de l'espace ; [...] l'univers un et multiple, est un effroi de plus pour l'homme, cette pauvre créature faite de trouble et de désordre. Il y a un écrasement entier de l'être, en face de la grande machine céleste qui fonctionne, muette et impassible, travaillant sans relâche à quelque labeur inconnu.

Je me souviens du frisson d'effroi que j'éprouvai lorsque notre maître de cosmographie nous parla, au collège, des abîmes du ciel. La pensée d'un tel infini, le nombre incalculable des mondes, les effrayantes distances de l'espace, toute cette machine céleste, écrasante pour la raison de l'homme dans son ordre souverain, ne peut entrer dans une cervelle humaine sans y causer une stupeur

12. Voir l'article de Michel Nathan, «La rêverie cosmique de Camille Flammarion », Romantisme, $\mathrm{n}^{\circ} 11,1976$, p. $75-85$.

13. Astronomie populaire, ouvr. cité, tome II, p. 549.

14. Ibid., tome I, p. 27-28. 
effarée. Un homme nerveux perdrait la tête à regarder trop longtemps le ciel, et se mettrait à hurler d'épouvante, comme une bête abandonnée dans la nuit ${ }^{15}$.

Non seulement l'anecdote personnelle devient un leitmotiv à cinq ans d'écart, mais elle l'exprime surtout avec ampleur au moyen d'une isotopie de la terreur et de la folie très hyperbolique. À peine l'embrayeur du souvenir est-il énoncé que l'auteur semble d'ailleurs "déserter» le texte, celui-ci évoquant la crise psychique provoquée par la vue des astres de façon abstraite, métonymique, la mettant ainsi à distance par une troisième personne à valeur plus rassurante. La dépossession de soi évoquée dans le souvenir se reproduit donc au plan de l'énonciation, confirmant un malaise évident du sujet zolien face à l'idée d'infini. La lecture même des ouvrages d'astronomie réitère chez Zola cette épreuve : "impression presque douloureuse ", " esprit éperdu ", "sensation de vertige ", "sentiment de panique " pour un écrivain qui voit alors « tout le poil de [sa] chair " s'hérisser. Les textes d'Amédée Guillemin ne suscitent pourtant en rien une telle lecture, rejoignant au contraire Flammarion dans l'idée d'un infini déployé comme une terre à conquérir pour l'homme :

Quel vaste champ, quel horizon magnifique le ciel n'ouvre-t-il pas à la plus active des facultés humaines, à l'imagination?

Étonnante puissance de l'homme! Enchaîné à la surface de la Terre, atome intelligent sur ce grain de sable perdu dans l'espace, il invente des appareils qui centuplent la pénétration de sa vue ; il sonde les profondeurs de l'abîme éthéré, jauge les dimensions de l'univers visible, et dénombre les myriades d'astres qui en peuplent l'effrayante étendue [...]. Telle est l'œuvre du génie et de la patiente persévérance des hommes qui se vouent depuis deux mille ans à l'étude des phénomènes dont le Ciel est le théâtre ${ }^{16}$.

Chez Flammarion, l'espace infini va même jusqu'à se muer en une allégorie de la vie toute-puissante très proche de celle que Zola appelle usuellement de ses vœux dans son art : "Oui, le ciel nous apparaît comme un immense laboratoire d'où l'inertie et l'immobilité sont exclues; ce n'est pas l'inactivité, ce n'est pas la mort qui règnent dans ses profondeurs, c'est la vie, immense, universelle, variée, toujours renaissante ${ }^{17}$ !» Inexplicablement, cette vision positive de l'infini semble totalement absente du discours zolien malgré son entière adhésion aux idées scientifiques défendues par Guillemin et Flammarion. L'écrivain évoque d'ailleurs incidemment le mouvement dysphorique de sa préhension de l'univers céleste dans cet unique exemple où il se distancie des propos d'A. Guillemin, sur la question de l'extinction du soleil :

M. Guillemin est consolant. Il ne croit pas que le Soleil s'éteigne de sitôt. [...] N'importe, bien que les savants nous assurent que l'astre n'est pas près de s'éteindre, je préférerais à tant de science, pour la paix de ma tête, l'heureuse ignorance du

15. L'Écho du Nord, art. cité, p. 322, et Le Gaulois, art. cité, p. 900.

16. Amédée Guillemin, Le Ciel. Notions d'astronomie à l'usage des gens du monde et de la jeunesse, Paris, Hachette, 1864, p. I et 1-2. Voir aussi Camille Flammarion, Astronomie populaire, ouvr. cité, tome I, p. 11-12 et tome II, p. 323.

17. Ibid., tome II, p. 476. 
laboureur qui aiguillonne tranquillement ses bœufs et qui regarde le Soleil comme un grand feu que Dieu a allumé là-haut et que les anges entretiennent pour mûrir ses moissons ${ }^{18}$.

Ce phénomène prend une tout autre ampleur dans l'univers de la fiction. Dans Les Rougon-Macquart, l'observation stellaire est paradoxalement associée à la remise en cause de toute intellection reposant sur un savoir, et donc de toute quête de la vérité.

\section{ITINÉRAIRE D’UN ASTRONOME AVEUGLE}

Dans Le Docteur Pascal, l'épisode du débat scientifique sous les étoiles est ainsi entièrement discrédité par la narration avant même de commencer. Le texte met tout d'abord Pascal en situation d'exercer sa posture d'herméneute convaincu : Clotilde a disparu, elle doit être dans le jardin et le scientifique part à sa recherche dans l'obscurité. Le comportement du médecin se pare alors de tous les signes d'un cheminement logique, invitant le lecteur à reconnaître sa démarche intellectuelle dans la quête de la jeune femme :

D'abord, il se pencha sur la balustrade de la terrasse, examina les pentes, les gradins de pierres sèches, qui descendaient jusqu'à la voie du chemin de fer ; mais rien ne remuait, il ne voyait que les têtes rondes et immobiles des petits oliviers. L'idée lui vint qu'elle était sans doute sous les platanes, près de la fontaine, dans le perpétuel frisson de cette eau murmurante ${ }^{19}$.

Ces premiers moments affichent une certaine maîtrise par un recours aux liens logiques, aux verbes de pensée, mais laissent bientôt place à une désorganisation manifeste :

Il y courut, il s'enfonça en pleine obscurité, une nappe si épaisse, que lui-même, qui connaissait chaque tronc d'arbre, devait marcher les mains en avant, pour ne point se heurter. Puis, ce fut au travers de la pinède qu'il battit ainsi l'ombre, tâtonnant, sans rencontrer personne. Et il finit par appeler, d'une voix qu'il assourdissait.

"Clotilde! Clotilde!»

La nuit restait profonde et muette. Il haussa peu à peu la voix.

"Clotilde! Clotilde!"

Pas une âme, pas un souffle. Les échos semblaient ensommeillés, son cri s'étouffait dans le lac infiniment doux des ténèbres bleues. Et il cria de toute sa force, il revint sous les platanes, il retourna dans la pinède, visitant la propriété entière ${ }^{20}$.

Aux liens logiques succède la parataxe : le docteur n'exécute plus un dessein, il ne fait qu'ajouter une action à une autre, sans la moindre réflexion. Pascal est impuissant, et devant cette faillite des outils rationnels, le savant est réduit à n'être qu'un corps, et encore, un corps qui lui échappe. L'homme accumule tous les symptômes de la panique

18. Le Gaulois, art. cité, p. 901.

19. Le Docteur Pascal, éd. citée, p. 987.

20. Ibid., p. 987-988. 
incontrôlable : il court, crie, se répète, interroge ; il en est réduit au tâtonnement, métaphore évidente d'une raison qui vacille. Dans ce contexte, l'obscurité du lieu devient symbolique de l'anéantissement de sa conscience. Au-delà de l'hyperbole qui la qualifie, la nuit ne se présente pas dans ce texte comme une absence de lumière, mais comme une présence, un monde du rien ayant une existence propre, signalée par les personnifications. Elle devient un espace qui absorbe Pascal grâce à ses frontières à sens unique ("s'enfonça en pleine obscurité "; " s'étouffait dans le lac des ténèbres "), sans jamais rien relâcher ("muette»). Le caractère violent de cette absorption ne fait aucun doute lorsqu'on regarde les verbes utilisés : l'étouffement, l'enfoncement suggèrent une mise à mort du sujet. Aveugle, inconscient, perdu, affolé, le médecin finit néanmoins par retrouver la jeune femme sur l'aire. Toutefois, la résolution de la quête ne renverse en rien la caractérisation du personnage :

Brusquement, il se trouva sur l'aire. [...] Pascal la traversait pour courir au verger, lorsqu'il manqua culbuter contre un corps, longuement étendu, qu'il n'avait pu voir. Il eut une exclamation effarée.

"Comment? Tu es là ${ }^{21}$ ?"

L'antéposition brutale de l'adverbe et la tournure pronominale figurent une sorte de télé-transportation : le personnage semble posé là, tombé du ciel, sans l'avoir commandé. Et c'est bien malgré lui que le héros du savoir résout sa quête. L'absence de vision avait réduit le personnage au pis-aller du tâtonnement, c'est-à-dire à une recherche désordonnée et empirique, par réaction au contact tactile; ici, ce n'est même plus le tâtonnement, mais la «culbute ": Pascal ne maîtrise plus son corps et c'est en dépit de lui qu'il « tombe " sur Clotilde. Encore cette découverte fortuite n'est-elle d'ailleurs pas présentée comme telle, car la femme est réifiée dans le texte comme une masse, celle d'un " corps qu'il n'avait pas pu voir ", qui ne désigne pas tant ici le corps biologique que le corps étranger, intrusif, menaçant, que l'esprit en déroute du médecin ne peut même plus rattacher à l'identité qui était pourtant le but de sa quête. Étrange traitement donc de ce héros zolien qui veut « ouvrir les yeux ", " tout connaître ${ }^{22}$ ". On ne peut dès lors que sourire devant l'ironie du débat qui suivra sous les étoiles, lorsque le docteur s'exclame : "Désormais, le repos dans l'ignorance est impossible. Aucune halte n'est à espérer, aucune tranquillité dans l'aveuglement volontaire. Il faut marcher, marcher toujours, marcher quand même, avec la vie qui marche toujours ${ }^{23}$. "Curieuse argumentation pour un herméneute aveugle qui peinait quelques minutes auparavant à mettre un pied devant l'autre! Le défenseur officiel de la connaissance objective du monde semble bien désavoué par l'univers romanesque qui l'entoure. 


\section{LE CIEL ÉTOILÉ, OU LA DESCRIPTION IMPOSSIBLE}

Une nuit étoilée qui par sa seule apparition fait crouler le discours " pascalien » de la foi en la science : la chose pourrait sembler étonnante, si elle ne se répétait dans l'ensemble du cycle des Rougon-Macquart. Dans Une page d'amour, l'observation des astres, occupant tout un chapitre, devient la mise en abyme du rapport des personnages au monde, un monde perçu comme incompréhensible et indicible, qui renvoie l'héroïne, Hélène, à sa méconnaissance d'elle-même depuis qu'elle essaie de refouler sa passion pour un homme marié. Face à la jeune veuve et à l'abbé Jouve, son confesseur, la description met en scène la disparition de la ville de Paris dans l'obscurité, avant de transformer le spectacle du ciel en un tableau artificiel et abstrait, permettant de mettre à distance la terreur que celui-ci semble inspirer. L'effacement du paysage urbain est tout d'abord présenté sur le mode de l'ajout, celui d'une couche de peinture recouvrant le fond d'un tableau : une "cendre fine ", " un flot d'encre " vient « ensevelir » la ville, l'« empli[r] » dans ses creux. Le noir n'est pas le rien, il est une surface visible qui a une existence matérielle. Les étoiles accèdent alors seulement à la visibilité :

Alors, elle leva les yeux, regardant le ciel d'été. Les constellations plantaient leurs clous d'or. Une planète, presque au ras de l'horizon, luisait comme une escarboucle, tandis qu'une poussière d'étoiles presque invisibles sablait la voûte d'un sable pailleté d'étincelles. Le Chariot, lentement, tournait, son brancard en l'air. [...] Derrière les milliers d'étoiles, d'autres milliers d'étoiles apparaissaient, et cela sans cesse, dans la profondeur infinie du ciel. C'était un continuel épanouissement, une braise attisée de mondes brûlant du feu calme des pierreries. La voie lactée blanchissait déjà, développait ses atomes de soleil, si innombrables et si lointains qu'ils ne sont plus, à la rondeur du firmament, qu'une écharpe de lumière ${ }^{24}$.

La comparaison inaugurale des "clous d'or" nous fait entrer dans le monde artificiel de l'artisanat et de l'ornement, instaurant d'emblée une recomposition humaine du référent étoilé. Faire d'une étoile un " clou planté », c'est aussi reconnaître que l'espace monochrome qui l'entoure n'est plus le ciel, mais une surface plane ayant une matérialité propre, sur laquelle vient s'apposer le point lumineux. C'est donc ôter par avance à la description toutes ses caractéristiques diégétiques propres pour la réduire à un élément factice et à une forme élémentaire. Le choix de cette métaphore est d'autant plus significatif qu'on la trouve aussi bien chez Guillemin que chez Flammarion, mais dans une utilisation radicalement opposée. L'image surgit en effet toujours chez les astronomes pour dire ce que n'est pas le ciel, et nier l'idée archaïque de "voûte " céleste :

Il n'est plus permis de les regarder comme des clous d'or fixés à une voûte solide et transparente ${ }^{25}$.

24. Émile Zola, Une page d'amour, Les Rougon-Macquart, éd. citée, tome II, p. 966.

25. Amédée Guillemin, Le Ciel, ouvr. cité, p. 1. 
Nous savons déjà que le ciel n'est pas une sphère concave sur laquelle des clous brillants seraient attachés, mais qu'il n'y a aucune espèce de voûte ; qu'un vide immense, infini, enveloppe la Terre de toutes parts ${ }^{26}$.

L'imagerie zolienne persiste donc dans une conception archaïque, dans une volonté manifeste d'artialiser l'espace infini du ciel pour mettre à distance son abîme par la substitution d'une paroi sécurisante faite de main d'homme. Or, la métaphore des "clous d'or" n'est pas appliquée aux étoiles en tant que telles dans le texte, mais à leurs " constellations ». La constellation est une figure abstraite, fictivement associée à un référent réel, de manière purement symbolique, sans autre légitimité que l'accord de la convention, que l'homme a plaquée sur le ciel pour mettre à distance l'autotélicité insupportable des lumières stellaires, à la fois fascinantes et effrayantes par leur contingence. Elle est un artefact que l'homme place devant les étoiles pour ne plus les voir vraiment. Pour ne plus sentir leur menace, il les réduit à des points, reliés arbitrairement par des lignes produisant des formes abstraites, permettant de les reconnaître, de les rendre visibles puis identifiables. Ce que cette métaphore transforme en une construction de clous n'est pas un élément naturel, mais ce qui était déjà conçu comme une abstraction du monde étoilé, signalant d'autant plus la difficulté zolienne de " dire » le ciel.

Le problème métaphysique soulevé par l'absence de référentialité des étoiles semble d'ailleurs pleinement pris en compte par Zola dans cet extrait. Toutes les images utilisées dans cette description ne font que gloser l'incapacité à décrire ou nommer l'ensemble de points faisant face à Hélène. Des tautologies montrent un embarras pour prédiquer le thème "étoiles ", comme si l'on ne pouvait qu'énoncer leur présence, sans les qualifier ; des oxymores - l'illimité dans l'unique, le visible invisible, le feu dans le calme - confirment ce caractère indicible ; des métaphores les associent à des formes minimales et immatérielles : des étoiles qui font apparaître des étoiles, des étoiles qui sablent le ciel de sable, des étoiles si nombreuses qu'elles sont presque invisibles, des étoiles semblables à de la poussière, à des atomes, à des étincelles... En somme, des étoiles qui ne ressemblent à rien et ne sont rien d'autres qu'elles-mêmes : des points, et encore, des points dont il est impossible de tirer un quelconque dessin figuratif en les assemblant. En faillite manifeste, la description se tourne alors vers la terre, où les lumières artificielles tirent peu à peu la ville du néant. Elle ne livre cependant aucune vision du paysage urbain, que le texte transforme en un prolongement du ciel étoilé :

Dans la mer de ténèbres, qui dormait devant eux, une étincelle avait lui. [...] Et, une à une, d'autres étincelles parurent. Elles naissaient dans la nuit avec un brusque sursaut, tout d'un coup, et restaient fixes, scintillantes comme des étoiles. Il semblait que ce fût un nouveau lever d'astres, à la surface d'un lac sombre. Bientôt elles dessinèrent une double ligne, qui partait du Trocadéro et s'en allait vers Paris, par légers bonds de lumière ; puis, d'autres lignes de points lumineux coupèrent celle-ci, des courbes s'indiquèrent, une constellation s'élargit, étrange et

26. Camille Flammarion, Astronomie populaire, ouvr. cité, tome II, p. 359. 
magnifique. Hélène ne parlait toujours pas, suivant du regard ces scintillements, dont les feux continuaient le ciel au-dessous de l'horizon, dans un prolongement de l'infini, comme si la terre eût disparu et qu'on eût aperçu de tous côtés la rondeur céleste ${ }^{27}$.

La description applique aux becs de gaz une métaphore filée stellaire unissant l'espace des lumières du sol à celui du ciel en une même surface. Les nouvelles " étoiles " partagent même, dans un deuxième niveau métaphorique, les comparants utilisés précédemment pour les vraies étoiles, comme les « étincelles». La suite de la description montre un accroissement de tous ces procédés, qu'elle applique également aux reflets des lumières dans la Seine, en faisant cette fois voisiner systématiquement les références astronomiques (termes techniques, noms de constellations) avec les toponymes des quartiers de Paris $^{28}$ : la ville se fait un substitut où l'auteur recrée à son gré un ciel artificiel et manipulable, entièrement créé de sa main à partir de la carte d'un monde connu. Cependant, la description semble d'elle-même dénoncer le stratagème de cette image supplétive en la désignant comme une réalisation graphique par le verbe "dessiner ». Le " dessin » décrit ne répond pourtant guère aux critères généralement admis comme siens : des lignes, coupées par d'autres lignes, des courbes... À part la forme elle-même, il n'y a à l'évidence rien à " voir " dans cette description abstraite et géométrisée, que le texte assimile avec un certain embarras à "une constellation étrange et magnifique ». Abstraction sur abstraction, la constellation est bien le seul comparant que l'écrivain peut en effet donner à ce qu'il vient de décrire, puisqu'elle-même est la solution que l'homme a trouvée pour donner un référent à ce qui n'en avait pas. Si ce n'est que cette nouvelle constellation «inventée " par Zola, personne n'en partage la convention, comme le souligne l'adjectif "étrange ». En quoi la constellation de Zola serait-elle étrange? Toutes ne sont que des amas de points reliés plus ou moins arbitrairement en fonction de leur proximité et de leur luminosité ; et les écrits de Flammarion ne se privent pas de les désigner comme telles $^{29}$. Ce que signale cet adjectif, ce n'est donc pas l'étrangeté de la constellation des lumières de Paris, mais le malaise que suscite chez l'écrivain toute constellation, révélant par sa contingence l'inconnu du ciel, la présence muette du réel, et par conséquent la situation aporétique d'un naturaliste qui se donnerait pour tâche de décrire le monde qui l'entoure.

27. Une page d'amour, éd. citée, p. 969.

28. «Leurs millions d'étoiles brûlaient avec un éclat fixe, dans une sérénité de nuit d'été », «en bas des pentes du Trocadéro, [...] une bande de comètes dont les queues d'or s'allongeaient en pluie d'étincelles », « la place de la Concorde, où luisait le scintillement d'une pléiade », "l'Esplanade [...] avec son rectangle de flammes, pareil à quelque Orion », « les quartiers populeux [...] luisant dans une confusion de nébuleuse », « une poussière qui emplissait les lointains de la ville de ces myriades de soleils, de ces atomes planétaires que l'œil humain ne peut découvrir », « une naissance de constellations » (ibid., p. 972-973).

29. Flammarion évoque « leurs figures plus ou moins étranges », tout en pointant leur aspect arbitraire : « il importe de concevoir que les positions des étoiles elles-mêmes, telles que nous les voyons, n'ont rien d'absolu, et que les configurations diverses qu'elles peuvent nous offrir ne sont qu' une affaire de perspective. [...] [La] disposition qu'elles revêtent à nos yeux n'est qu'une apparence causée par la position de la Terre vis-à-vis d'elles » (Astronomie populaire, ouvr. cité, tome II, p. 359). 
Et de remise en cause du référent il est bien question tout au long de ce chapitre, qui met en abyme cette inadéquation entre ce qui est vu et ce qui est su, compris, identifié. En bonne herméneute, Hélène s'enquiert en effet auprès de l'abbé du nom des étoiles qu'elle a sous les yeux :

«Tenez, dit-elle à son tour, cette petite étoile bleue, dans ce coin du ciel, je la retrouve tous les soirs..." [...]

À deux reprises, elle le questionna sur des noms d'étoiles; toujours la vue du ciel l'avait tourmentée. Mais il hésitait, il ne savait pas.

"Vous voyez, demandait-elle, cette belle étoile qui a un éclat si pur?

— À gauche, n'est-ce pas? disait-il, près d'une autre moins grosse, verdâtre... Il y en a trop, j'ai oublié."

Ils se turent, les yeux toujours levés, éblouis et pris d'un léger frisson en face de ce fourmillement d'astres qui grandissait. [...]

"Cela me fait peur ", dit Hélène à voix très basse.

Et elle pencha la tête pour ne plus voir ${ }^{30}$.

Les interrogations d'Hélène tentent désespérément de faire coïncider le voir avec l'intelligible, le déictique qu'elle montre du doigt avec un identifiable, comme le montre l'inflation des tournures phatiques et des démonstratifs dans son discours. Mais cette volonté rencontre une altération du lien nom/référent : l'abbé se révèle incapable de les faire coïncider, et le narrateur ne vient pas même réparer sa défaillance pour indiquer au lecteur quelle peut bien être la chose que les personnages voient, bien que la description sache par ailleurs évoquer le Chariot ou Orion... Il y a donc une volonté manifeste de ne pas identifier les formes qu'Hélène a sous les yeux, de contrecarrer tout regard herméneutique. Le rendement descriptif de cette place accordée à la seule perception s'avère en outre très négligeable. Quel est le contenu de la vision d'Hélène ? Des points, laissant peu de pouvoir à la parole descriptive, et d'ailleurs si peu que les deux personnages ont bien du mal à se comprendre, à distinguer l'étoile dont l'un parle à l'autre. Il est en effet impossible de décrire longuement des étoiles sans passer dans le monde de la constellation, de l'identification. Refuser cette codification, c'est revenir à la forme de l'étoile même, c'est-à-dire à l'unité minimale, au rien de la description et du dessin : le point. Le lecteur à l'imagination la plus fertile ne trouvera rien de plus à se figurer dans cette scène que trois points lumineux sur un fond sombre, un bleu, un blanc et un verdâtre, et non une belle nuit d'été.

Les difficultés de la description à dire le ciel, la recréation d'une constellation " urbaine » faite de lumières artificielles et l'impossible identification des étoiles par les personnages manifestent un réel trouble lié aux astres dans ce roman, qui rappelle les anecdotes utilisées par Zola dans ses articles sur A. Guillemin. Les questions qu'Hélène formule sur le nom des étoiles s'apparentent à un moyen de dépasser la peur que celles-ci provoquent en elle, de les mettre à distance par une illusion d'emprise rationnelle. Le personnage répète donc l'état primitif des civilisations ayant cartographié le ciel pour en exorciser l'inconnu, démarche que la description zolienne

30. Une page d'amour, éd. citée, p. 966-967. 
applique aussi à son tour sans toutefois parvenir à imposer "son " ciel à la place de celui qu'elle ne peut maîtriser.

Les ciels nocturnes d'Une page d'amour, mais aussi de L'Euvre dont les descriptions réitèrent les mêmes phénomènes face au peintre Claude Lantier, s'apparentent ainsi à des préfigurations outrageusement radicales des nuits de Van Gogh - qui a beaucoup lu Zola et Flammarion. Géométriques, sans référent, les étoiles chez Zola sont toujours vues sur le mode de la constellation, c'est-à-dire d'une recomposition formelle tentant de redonner à l'indicible étoilé une " figure ", une forme permettant de l'appréhender. Si ce n'est que les constellations zoliennes n'ont guère de forme identifiable, et se transforment en étranges dessins abstraits, impossibles à nommer. Se manifeste ainsi chez l'écrivain un rapport dysphorique à l'observation du ciel, devenu l'emblème d'une impossible maîtrise du monde. Le parasitage du discours théorique sur l'astronomie par la disqualification de ses relais dans la fiction ne remet pas en cause l'intérêt de Zola pour l'infini étoilé, mais le replace dans une relation de fascination/répulsion qui remet en cause la préhension du réel sur laquelle se fonde toute l'esthétique naturaliste. Cette mise en image du ciel très particulière témoigne, pour un Zola écrasé par son incapacité à dire le monde, d'un besoin de formalisation, de mise à distance de la brutalité du réel, suscité par la vision de l'inconnu des étoiles. Le peintre réaliste de L'Euvre, Claude, qui se refusera à cette formalisation et se perdra dans son désir d'observer encore et toujours, finira d'ailleurs par se suicider au lendemain de cette contemplation.

Le rapport de Zola à la diffusion de la culture astronomique au XIX ${ }^{\mathrm{e}}$ siècle se révèle intéressant dans son incohérence, à valeur d'exemple et de contre-exemple : amateur éclairé, l'écrivain utilise les progrès de l'astronomie comme un moteur de sa campagne théorique pour la vérité et la victoire du progrès scientifique sur l'obscurantisme ; cependant, cette quête exaltée se révèle n'être que le paravent trop décoré d'un observateur inquiet du ciel cristallisant son rapport traumatique au réel, qu'il tente désespérément de maîtriser par un recours à l'abstraction, filtrant l'observation : la géométrie abstraite, donc, contre la terreur inspirée par la vision de l'univers au travers de la lunette astronomique, dans une désincarnation bien lointaine de la «viande " décriée par Flammarion. 